\title{
THE NUMBER OF UNLABELLED GRAPHS WITH MANY NODES AND EDGES
}

\author{
BY E. M. WRIGHT ${ }^{1}$
}

Communicated by G.-C. Rota, May 1, 1972

$T=T(n, q)$ is the number of graphs with $n$ unlabelled nodes and $q$ undirected edges, each pair of different nodes being not joined or joined by a single edge. We write $N=n(n-1) / 2$, so that a graph contains at most $N$ edges. We give here asymptotic approximations to $T$ for all large $n$ and $q$. Since $T(n, q)=T(n, N-q)$, we suppose $q \leqq N / 2$ throughout.

In what follows, $A, C$ and $\eta$ denote numbers, not always the same at each occurrence. Of these, $A$ and $C$ are positive and independent of $n$ and $q$. A number $A$ denotes any positive number that we may choose, while $C$ is a suitable positive number which may depend on any $A$ present or implied. Unless we specifically state the contrary, all our statements carry the implied condition that $q>C$ and $n>C$. The $O$-notation refers to the passage of $n$ and $q$ to infinity and the constant implied is a $C$. An $\eta$ is any number which is $O\left(q^{-C}\right)$ for some $C$.

We write

$$
\begin{array}{ll}
B(h, k)=h ! /\{k !(h-k) !\}, & \Lambda_{n}=\Lambda(n, q)=B\{n(n-1) / 2, q\} / n !, \\
\mu=(2 q / n)-\log n, & J(v)=v^{1 / 2}\{2(1+\log v)\}^{-1 / 2}, \\
\delta=\mu J(n), & K(v)=2 \pi^{1 / 2} e^{-1} J(v), \\
\text { Erf } x=2 \pi^{-1 / 2} \int_{0}^{x} e^{-t^{2}} d t, & \lambda(x)=(1+\operatorname{Erf} x) / 2 .
\end{array}
$$

A table of $\operatorname{Erf} x$ is given in [1]. We write $V$ to denote the greatest integer such that $V \log V \leqq 2 q$.

Polya [2] proved that $T \sim \Lambda_{n}$ as $n, q \rightarrow \infty$, provided that $(N / 2)-$ $q<A n$, and Oberschelp [5] weakened the condition to $(\mathrm{N} / 2)-q<\mathrm{Cn}^{3 / 2}$, In $[8]$, I proved the following theorem.

THEOREM 1. The necessary and sufficient condition that $T \sim \Lambda_{n}$ is that $\mu \rightarrow \infty$ as $n \rightarrow \infty$. If this condition is satisfied, then $T=\Lambda_{n}\left\{1+O\left(e^{-C \mu}\right)\right\}$.

Korsunov [4] stated the following theorem without proof.

AMS 1969 subject classifications. Primary 0565

Key words and phrases. Unlabelled graphs, asymptotic enumeration.

1 The research reported herein was sponsored in part by the United States Government. 
THEOREM 2. If $\mu>C$ then

$$
T \sim \Lambda_{n} \exp \left(-e^{-\mu}\right) /\left(1-e^{-\mu}\right) .
$$

In [9], I stated results which included.

THeORem 3. If $\mu<-A n^{-1 / 2} \log n$, then $T \sim K(V) A_{V}$.

The asymptotic approximation to $T$ in Theorem 3 is independent of $n$. This is the asymptotic analogue of the fact that $T(n, q)=T(2 q, q)$ for all $n \geqq 2 q$,

There is an obvious gap between the conditions of Theorems 2 and 3 . Here I fill this, but the results are a little more complicated. Again for applications we require rather more accurate information than that given in Theorems 2 and 3; we must replace the implied factor $1+o(1)$ by $1+\eta$. Theorems 2 and 3 are replaced by the following theorems.

THEOREM 4. If $\mu>A n^{(A-1) / 2}$, then

$$
T=\Lambda_{n} \exp \left(-e^{-\mu}\right)\left(1-e^{-\mu}\right)^{-1}(1+\eta) .
$$

THEOREM 5. If $\mu<-A n^{-1 / 2} \log n$, then $T=K(V) \Lambda_{V}(1+\eta)$.

The next two theorems are best understood if we remark that

$$
\lambda(-x)=\left\{e^{-x^{2}} /(2 x)\right\}\left(1+O\left(x^{-2}\right)\right)
$$

if $x$ is large and positive and that $\lambda(x) \rightarrow 1$ as $x \rightarrow+\infty$.

THEOREM 6. If $\mu<A n^{-1 / 2}(\log n)^{1 / 2}$, so that $\delta<A$, then

$$
T=\lambda(-\delta) K(V) \Lambda_{V}(1+\eta) .
$$

THEOREM 7. If $0 \leqq \mu \leqq n^{-1 / 4}$, then

$$
T=K(n) e^{\delta^{2}} \lambda(-\delta) \Lambda_{n}(1+\eta) .
$$

The intervals of values of $q$ in the hypotheses of our theorems now overlap. From our results we can deduce the following two theorems which we shall find useful in applications, especially to the improvement of the result in [10] about the probability of connectedness of an unlabelled graph. We write $R(n, q)=T(n, q+1) / T(n, q)$.

THEOREM 8. If $n \log n \leqq 2 q \leqq N-2$, then

$$
R(n, q)=(N-q)(q+1)^{-1}\left\{1+O\left(\min \left(\eta, e^{-C \mu}\right)\right)\right\} .
$$

If $C<2 q \leqq n \log n$, then $R(n, q)=\left(V^{2} / 2 q\right)(1+\eta)$.

THEOREM 9. If $n>C$ and $1 \leqq q \leqq(N / 2)-1$, then $T(n, q+1)>$ $T(n, q)$. 
In Theorem 9 alone we set aside the condition $q>C$ implied elsewhere. The result of Theorem 9 is false for $n=5$, when $N=10$ and $T(5,4)$ $=T(5,5)=6$, but it is true (from the tables [6]) for $6 \leqq n \leqq 18$. One would expect it to be true for all $n \geqq 6$, but I cannot prove this.

Korsunov [4] extends his result (Theorem 2 above) with appropriate modifications to other classes of graphs. All my results here can be similarly extended, for example, to the eight different classes considered in [3] and [7], but I confine myself to the one class of graphs for simplicity.

\section{REFERENCES}

1. L. J. Comrie, Chambers' six-figure math. tables. II, Table XIIA, 1963, pp. 518-519.

2. G. W. Ford and G. E. Uhlenbeck, Combinatorial problems in the theory of graphs. IV, Proc. Nat. Acad. Sci. U.S.A. 43 (1957), 163-167. MR 19, 113.

3. E. N. Gilbert, Enumeration of labelled graphs, Canad. J. Math. 8 (1956), 405-411. MR 18, 408.

4. A. D. Korsunov, On the power of some classes of graphs, Dokl. Akad. Nauk SSSR 193 (1970), 1230-1233 = Soviet Math. Dokl. 11 (1970), 1100-1104.

5. W. Oberschelp, Kombinatorische Anzahlbestimmungen in Relationen, Math. Ann. 174 (1967), 53-78. MR 36 \# 1342.

6. M. L. Stein and P. R. Stein, Enumeration of linear graphs and connected linear graphs up to $P=18$ points, Los Alamos Scientific Laboratory, 1963 .

7. E. M. Wright, Asymptotic enumeration of connected graphs, Proc. Roy. Soc. Edinburgh Sect. A 68 (1970), 298-308. MR 42 \#1723.

8. - Graphs on unlabelled nodes with a given number of edges, Acta Math. 126 (1971), 1-9. MR 42 \# 2975.

9., Unlabelled graphs with many nodes and edges, Notices Amer, Math. Soc. 19 (1972), A-3. Abstract \# 72T-49.

10. - The probability of connectedness of an unlabelled graph can be less for more edges, Proc. Amer. Math. Soc. 35 (1972), 21-25.

Office of the Principal, University of Aberdeen, Aberdeen, Scotland 